\title{
Modificaciones de curva velocidad-tiempo de la cadera en el estilo braza a distintas velocidades de nado
}

J.J. MERCADÉ, R. ARELLANO, V.M. SOTO.

Facultad de Ciencias de la Actividad Física y el Deporte. Universidad de Granada

\begin{abstract}
Resumen
El propósito del presente estudio fue observar cual era el comportamiento de la curva de velocidad de la cadera durante el nado estilo braza a diferentes velocidades de nado. Dos nadadoras de distinto nivel fueron seleccionadas para participar en el presente estudio en el que realizaron cuatro repeticiones de 25 m a velocidad diferente. Dos cámaras de vídeo PAL, una subacuática y otra exterior, fijas y perpendiculares a la dirección de desplazamiento del nadador, se emplearon para analizar un ciclo completo de nado de cada una de las repeticiones de nado. Se calcularon velocidades promedio de nado, velocidades máximas intra-ciclo, velocidades mínimas intra-ciclo, frecuencias, longitudes y duración de ciclo. Se encontró que al incrementar la velocidad promedio de nado, los valores máximos intra-ciclo aumentaban, no observándose modificación alguna en el valor mínimo. Además se observó un aumento de la frecuencia de ciclo y una disminución de la longitud de ciclo a medida que la velocidad se incrementaba, debido a la reducción del tiempo de deslizamiento tras la patada.
\end{abstract}

Palabras Clave: Natación, Braza, Fotogrametría 2-D, Velocidad.

\section{Introducción}

Gran cantidad de investigaciones en natación han centrado su interés en el estudio biomecánico de un ciclo completo de nado en cada uno de los estilos. Observar la interacción existente entre las fuerzas propulsivas y las resistivas durante un ciclo es de gran relevancia a la hora de determinar el nivel de destreza de un nadador (D'Acquisto y Costill, 1998). Una correcta aplicación de las fuerzas propulsivas sobre el agua, así como una reducción de las resistencias que se oponen al avance del nadador, determinan la velocidad de desplazamiento en el medio acuático (Maglischo, 1987; Costill y D'Acquisto, 1987; Maglischo, 1987; Chengalur y Brown, 1992; D’Acquisto y Costill, 1998). La interacción entre ambas genera fluctuaciones de la velocidad intra-ciclo (Kreighbaum y Barthels, 1990). Cuanto más uniforme sea la velocidad durante todo el transcurso del ciclo de nado, menor será el gasto energético (Vilas-Boas y Santos, 1994; Vilas-Boas, 1996).

La braza se caracteriza por ser el estilo que mayores fluctuaciones de la velocidad intra-ciclo presenta (Craig y Pendergast, 1979; Maglischo, 1993), debido a una mayor oposición del medio al avance del cuerpo en forma de resistencias
(Kolmogorov y col., 1997). Por un lado, se producen incrementos de la velocidad horizontal (linear) durante las fases propulsivas de ambos brazos y piernas; y, por el contrario, en las fases no propulsivas, recobro de brazos y piernas, resulta en un descenso de la velocidad linear (Kent y Atha, 1975; D'Acquisto y Costill, 1988; D'Acquisto y Costill, 1998; Chollet y col., 1999). Dentro de las variantes de nado a estilo braza (ondulatoria y plana), la ondulatoria es la que presenta menores fluctuaciones de la velocidad intra-ciclo, y por lo tanto mayor economía energética (Tilborgh, 1988; Colman y Persyn, 1991).

La particular fluctuación de la velocidad intraciclo en el estilo braza parece variar con la velocidad de nado (Manley y Atha, 1992). Al incrementarse la velocidad de nado los picos de velocidad máximo y mínimo aumentan, pero en menor cuantía este último, debido a una mayor aplicación de fuerzas propulsivas y a un descenso en el tiempo de la fase de deslizamiento entre ambas acciones propulsivas (Chollet y col., 1999).

El objetivo del presente estudio fue observar cual era el comportamiento de la curva de velocidad de la cadera durante el nado estilo braza a diferentes velocidades de nado. 


\section{Método}

Dos nadadoras, ambas de 13 años de edad, y distinto nivel de rendimiento (sujeto A 96,21 s. y sujeto B $82,71 \mathrm{~s}$. en la prueba de $100 \mathrm{~m}$. braza) fueron seleccionadas para el presente estudio, previo consentimiento de las mismas.

El estudio se llevo a cabo en una piscina de 6 calles de $12,5 \mathrm{~m}$ de anchura y $25 \mathrm{~m}$ de longitud. Para la toma de las distintas secuencias de nado, se emplearon dos cámaras de video SONY sistema PAL de $8 \mathrm{~mm}$ de cinta y objetivo de $46 \mathrm{~mm}$, situadas a igual distancia de ambas paredes $(12,5$ $\mathrm{m})$, sirviendo una de ellas de visión subacuática (tiempo de obturación 1/250) y otra de visión exterior (tiempo de obturación 1/1000). Ambas cámaras se encontraban conectadas a una mezcladora de vídeo (Panasonic Digital AV Mixer WJ-AVES) empleada para simultanear en un monitor de TV (SONY 14 pulgadas color Trinitron) las imágenes procedentes de ambas cámaras, así como los códigos de tiempo insertados a través de un video cronómetro. Todas las secuencias de nado fueron almacenadas en cintas de vídeo VHS por medio de un magnetoscopio (SONY VHS SLV-777NC).

Ambos sujetos realizaron cuatro series de $25 \mathrm{~m}$ con recuperación activa $(25 \mathrm{~m}$.) y pasiva $(90 \mathrm{~s}$.) incrementando la velocidad de una serie a otra. De cada una de las series se analizó un ciclo completo de nado, llevándose a cabo el proceso de tratamiento de datos y generación de resultados en el Laboratorio del Movimiento Humano de la Facultad de Ciencias de la Actividad Física y del Deporte de Granada, utilizando el sistema de análisis fotogramétrico CIBORG 3.0 (Soto, 1995).

Para conocer la velocidad linear del centro de gravedad (CG) se digitalizó fotograma a fotograma un punto situado a la altura de la cadera. Como recoge Maglischo y col. (1987), la velocidad linear medida a la altura de la cadera no difiere significativamente con respecto a la velocidad del centro de masas. Varios estudios comparativos entre el CG y la cadera, indican que las fluctuaciones de la velocidad intra-ciclo son más extremas para éste último punto que para el CG (Maglischo y col., 1987; Costill y col., 1987; Mason y col., 1989). Por ejemplo, la velocidad registrada en el punto de la cadera al final de la fase de recobro de piernas es de $0.4 \mathrm{~m} / \mathrm{s}$; mientras que los valores de velocidad registrados para el CG en el mismo instante ronda los $0.6 \mathrm{~m} / \mathrm{s}$. Así mismo la cadera, con respecto al $\mathrm{CG}$, alcanza mayores valores de velocidad durante la fase propulsiva de la patada.
Para profundizar algo más en lo que sucede en el centro de gravedad (cadera) durante el ciclo de nado, se subdividió el ciclo en cuatro fases. La primera de ellas se prolonga desde el inicio del ciclo (momento de máxima flexión de rodilla durante el recobro de piernas) hasta el primer pico de velocidad máxima horizontal, correspondiente con la finalización del recobro de brazos y finalización del movimiento de pies hacia huera. La segunda fase comprende desde el primer pico de velocidad horizontal máxima hasta el pico de velocidad mínima que se genera entre ambos picos máximos, correspondiente a la fase de transición entre la acción propulsiva de piernas y de brazos. La tercera se inicia desde la momento de finalización de la fase anterior hasta el instante en que se alcanza el segundo pico de velocidad máxima, que coincide con el movimiento hacia dentro de las manos. Por último, la cuarta fase se extiende desde el momento de finalización de la fase anterior hasta que de nuevo las rodillas alcanzan su grado de máxima flexión (fin del ciclo).

\section{Resultados}

Una vez obtenida la curva de velocidad horizontal de la cadera durante un ciclo completo de nado en cada una de las repeticiones realizadas por ambos sujetos (Figuras 1 y 2), se extrajeron los siguientes datos: valor máximo y mínimo de velocidad intra-cíclica horizontal, expresados en metros por segundo; duración de ciclo (s.); longitud de ciclo $(\mathrm{m})$; velocidad promedio de nado $(\mathrm{m} / \mathrm{s})$; frecuencia de ciclo (Hz.); y, por último, la duración en el tiempo (s.) de cada una de las fases en que se subdividió el ciclo de nado (Fases 1 a 4). Los valores numéricos de cada uno de los factores de análisis se recogen en la Tabla 1.

En las Figuras 1 y 2 se observa como al aumentar la velocidad promedio de nado se incrementa el pico máximo de velocidad intra ciclo, alcanzando los Sujetos A y B en la repetición más rápida valores de 1,695 y $1,599 \mathrm{~m} / \mathrm{s}$, respectivamente. En cuanto al pico mínimo de velocidad intra-ciclo no se observa ningún comportamiento estandarizado, rondando por termino medio los $0.087 \mathrm{~m} / \mathrm{s}$ en el Sujeto A y $0,25 \mathrm{~m} / \mathrm{s}$ en $\mathrm{B}$.

Entrando en el análisis del ciclo por fases, al incrementar la velocidad promedio de nado se reduce la duración total del ciclo (Figuras 1 y 2). Aunque no se observe un comportamiento estándar en ambas nadadoras en cuanto a la duración cada una de las fases constituyentes del ciclo, si unimos la 
segunda y tercera fase, que podría considerarse como el tiempo transcurrido entre la acción propulsiva de piernas y brazos (fase de deslizamiento), se denota una disminución en su duración a medida que se incrementa la velocidad promedio de nado. Traduciéndose esto en valores numéricos, en el Sujeto A se reduce de un 63,5 a un 44,1\% respecto a la duración total del ciclo, es decir, de
1,46 a 0,63 s.; y, en el Sujeto B, de un 63,8 a un 46,4 $\%$ (1,18 a $0,51 \mathrm{~s})$. En las Figuras 3 y 4 se representa gráficamente el comportamiento de la frecuencia y longitud de ciclo al incrementarse la velocidad. En ambos sujetos se observa una tendencia a disminuir la longitud e incrementar la frecuencia de ciclo a medida que se suceden cada una de las repeticiones de las que consta el estudio.

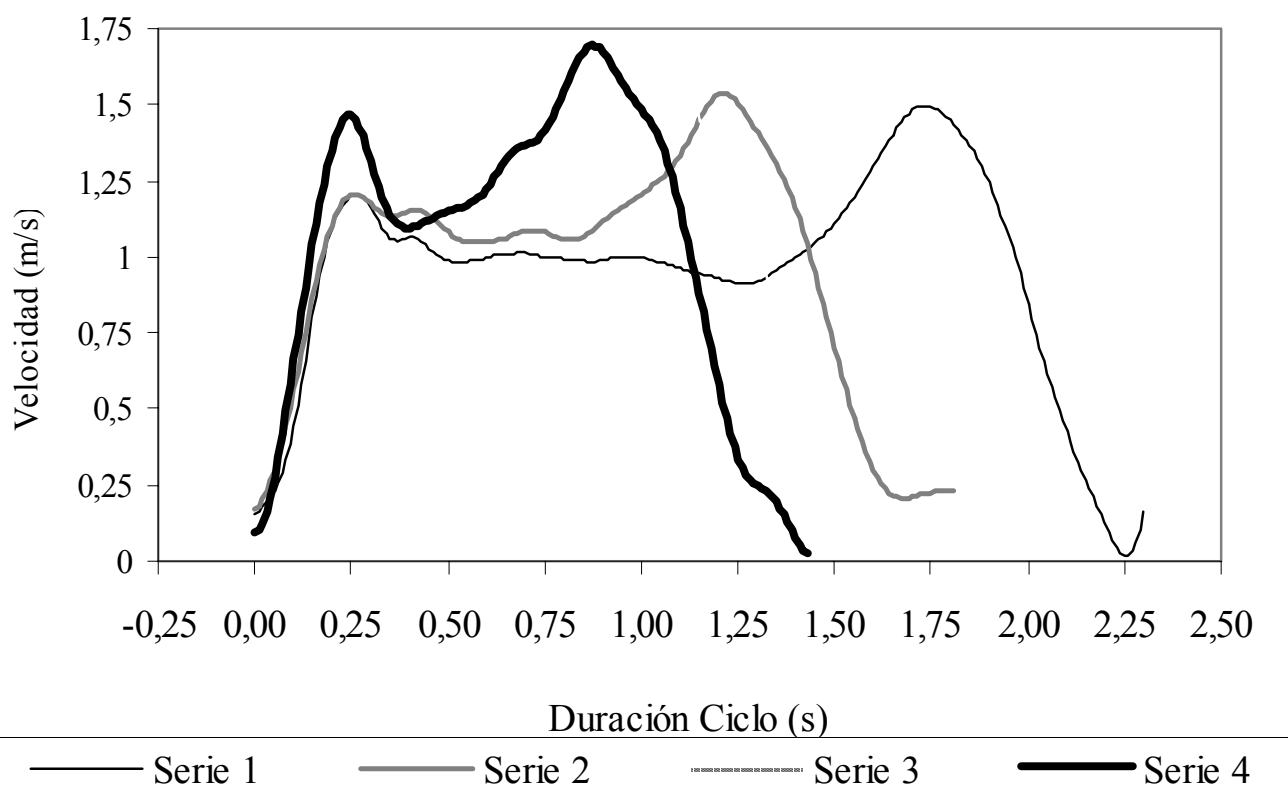

Figura 1. Comportamiento de la curva de velocidad de la cadera en el sujeto A.

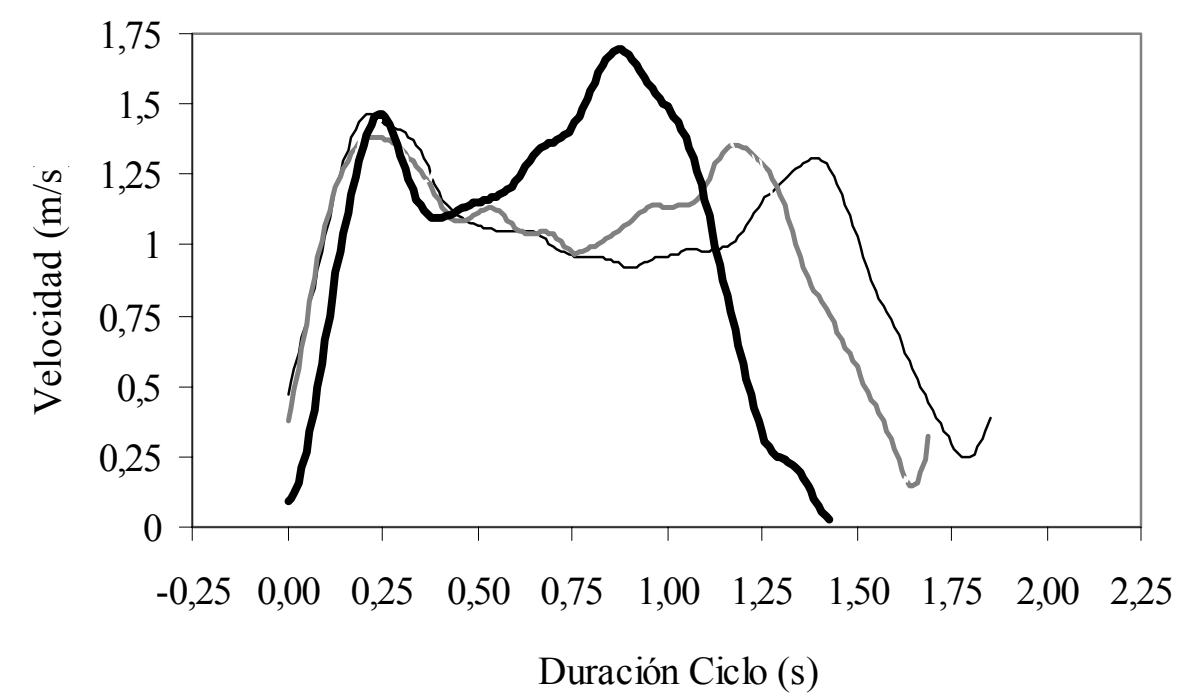

\begin{tabular}{|lll|}
\hline Serie 1 & Serie $2 \quad$ Serie $3 \quad$ Serie 4 \\
\cline { 2 - 2 }
\end{tabular}

Figura 2. Comportamiento de la curva de velocidad de la cadera en el Sujeto B. 


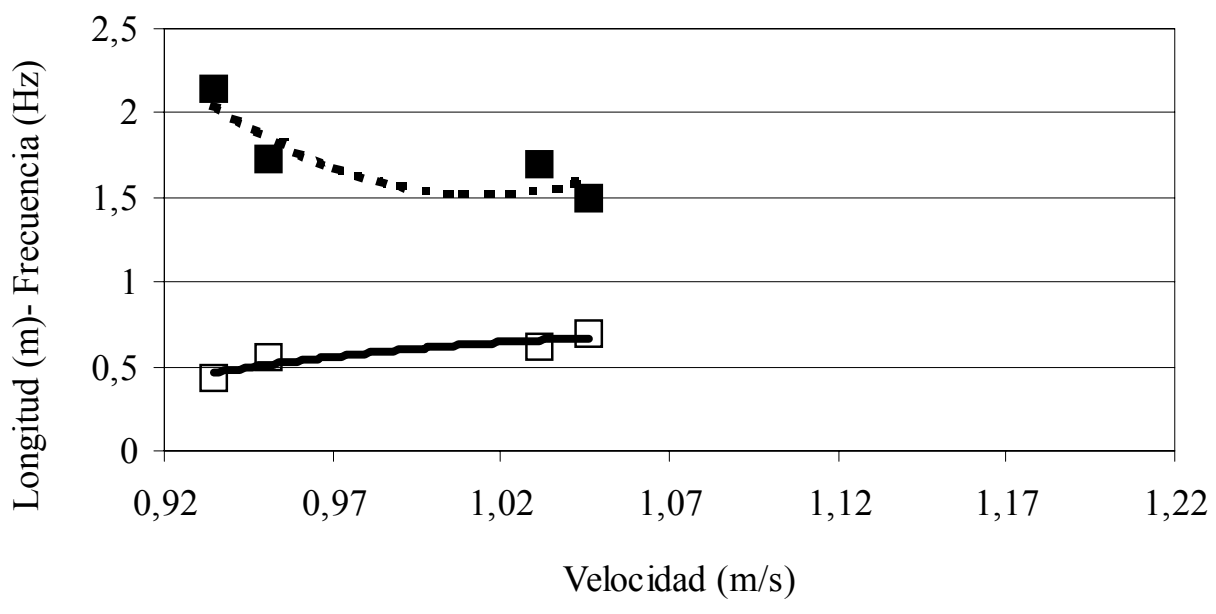

\section{LC $\square$ FC - - Polinómica (LC) —Polinómica (FC)}

Figura 3. Variaciones de la longitud (m) y frecuencia de ciclo (Hz.) en el nado del Sujeto A al incrementar la velocidad promedio de nado.

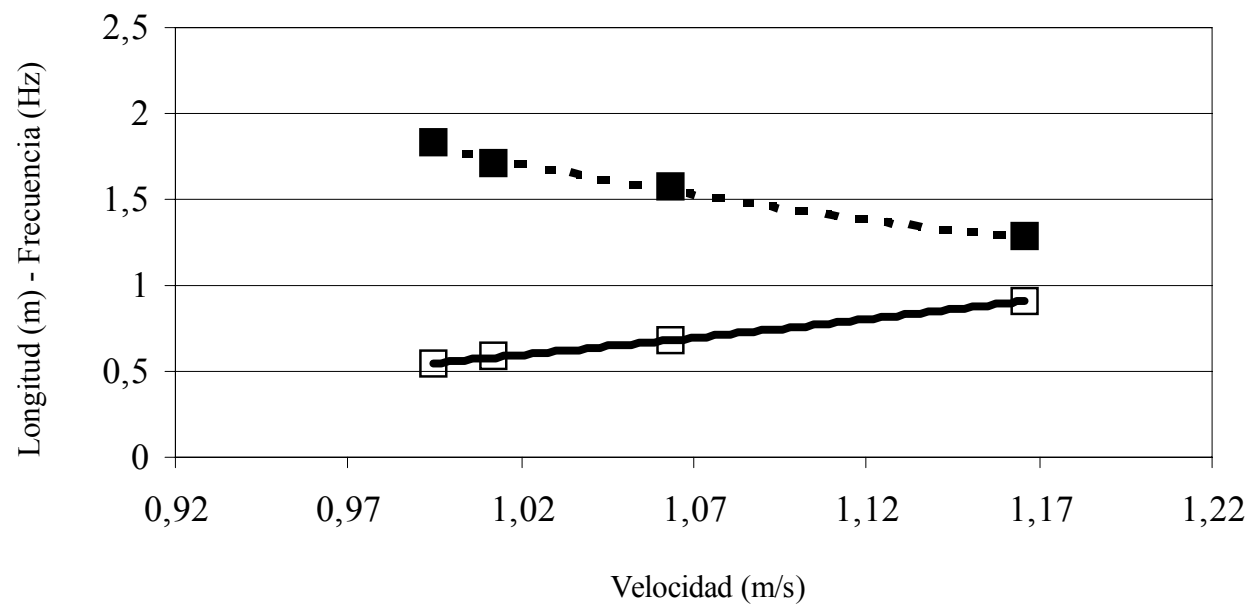

\section{LC $\square$ FC - - Polinómica (LC) —Polinómica (FC)}

Figura 4. Variaciones de la longitud (m) y frecuencia de ciclo (Hz.) en el nado del Sujeto $\mathrm{B}$ al incrementar la velocidad promedio de nado.

Tabla 1. Valores numéricos de cada uno de los factores analizados en el presente estudio.

\begin{tabular}{|c|c|c|c|c|c|c|c|c|c|c|c|c|}
\hline $\begin{array}{c}\mathrm{S} \\
\mathrm{U} \\
\mathrm{J} \\
\mathrm{E} \\
\mathrm{T} \\
\mathrm{O}\end{array}$ & $\begin{array}{l}\mathrm{S} \\
\mathrm{E} \\
\mathrm{R} \\
\mathrm{I} \\
\mathrm{E}\end{array}$ & $\begin{array}{c}\text { Velocidad } \\
\text { Nado } \\
(\mathrm{m} / \mathrm{s})\end{array}$ & $\begin{array}{c}\text { Velocidad } \\
\text { Horizontal } \\
\text { Máxima } \\
(\mathrm{m} / \mathrm{s})\end{array}$ & $\begin{array}{l}\text { Velocidad } \\
\text { Horizontal } \\
\text { Mínima } \\
(\mathrm{m} / \mathrm{s})\end{array}$ & $\begin{array}{c}\text { Tiempo } \\
\text { Ciclo } \\
\text { (s) }\end{array}$ & $\begin{array}{l}\text { Longitud } \\
\text { Ciclo }(\mathrm{m})\end{array}$ & $\begin{array}{l}\text { Frecuencia } \\
\text { Ciclo }(\mathrm{Hz})\end{array}$ & $\begin{array}{c}\text { FASE } \\
1(s)\end{array}$ & $\begin{array}{c}\text { FASE } \\
2(s)\end{array}$ & $\begin{array}{c}\text { FASE } \\
3(\mathrm{~s})\end{array}$ & $\begin{array}{c}\text { FASE } \\
4(\mathrm{~s})\end{array}$ & $\begin{array}{c}\text { FASE } \\
2+3 \\
\text { (s) }\end{array}$ \\
\hline \multirow{4}{*}{ A } & $1^{\circ}$ & 0,935 & 1,498 & 0,019 & 2,30 & 2,151 & 0,435 & 0,27 & 0,99 & 0,47 & 0,47 & 1,46 \\
\hline & $2^{\circ}$ & 0,951 & 1,536 & 0,167 & 1,81 & 1,721 & 0,552 & 0,26 & 0,31 & 0,64 & 0,64 & 0,95 \\
\hline & $3^{\circ}$ & 1,031 & 1,661 & 0,137 & 1,64 & 1,691 & 0,610 & 0,24 & 0,38 & 0,45 & 0,45 & 0,83 \\
\hline & $4^{\circ}$ & 1,046 & 1,695 & 0,026 & 1,43 & 1,496 & 0,699 & 0,24 & 0,16 & 0,47 & 0,47 & 0,63 \\
\hline \multirow{4}{*}{ B } & $1^{\circ}$ & 0,995 & 1,383 & 0,250 & 1,85 & 1,840 & 0,541 & 0,22 & 0,69 & 0,49 & 0,49 & 1,18 \\
\hline & $2^{\circ}$ & 1,012 & 1,463 & 0,145 & 1,69 & 1,710 & 0,592 & 0,24 & 0,52 & 0,42 & 0,42 & 0,94 \\
\hline & $3^{\circ}$ & 1,063 & 1,579 & 0,297 & 1,48 & 1,573 & 0,676 & 0,18 & 0,5 & 0,38 & 0,38 & 0,88 \\
\hline & $4^{\circ}$ & 1,166 & 1,599 & 0,333 & 1,10 & 1,283 & 0,909 & 0,21 & 0,26 & 0,25 & 0,25 & 0,51 \\
\hline
\end{tabular}




\section{Discusión y conclusiones}

Las fluctuaciones de la velocidad existentes durante un ciclo completo de nado estilo braza, se deben a las características coordinativas de este estilo, es decir, a la aplicación intermitente de fuerzas propulsivas, y a los cambios continuos en la posición de brazos, piernas y tronco (Kent y Atha, 1971; Kent y Atha, 1975; Craig y Pendergast, 1979; Costill y col., 1987; D’Acquisto y col., 1988; Maglischo, 1993). Grandes fluctuaciones de la velocidad intraciclo conlleva un mayor gasto energético (VilasBoas, 1996).

Las fases que componen el ciclo de nado estilo braza no se ven afectadas de la misma forma al incrementarse la velocidad promedio de nado (Sanders, 1996). La reducción significativa del tiempo de deslizamiento hallada en este estudio, en relación al incremento de la velocidad, se halla en concordancia con los resultados obtenidos en estudios precedentes (Soares y col., 1999). Los datos numéricos recogidos en el presente estudio entorno a la duración del ciclo, se encuentran dentro del intervalo de 1,05 s. y 2,2 s. recogidos por Klauck y col. (1990) y Craig y col. (1988), respectivamente, para distintos ritmos de nado.

El aumento en la velocidad promedio de desplazamiento no produce modificación alguna del pico mínimo de velocidad intra-ciclo, pero al igual que lo hallado por Manley y Atha (1992), sí se produce un incremento del pico máximo de velocidad intra-ciclo. Comparando con otros estudios que analizan ciclos de nado desarrollados a velocidad máxima y tomando como punto de análisis la cadera, encontramos que los valores de velocidad máxima intraciclo registrados en ambas nadadoras son similares a los 1,64 m/s de media obtenidos por D' Acquisto y Costill (1998) en un grupo de nadadoras norteamericanas. Este incremento de la velocidad máxima intra-ciclo puede explicarse por una mayor aplicación de fuerza por parte de las superficies propulsoras, además de lo ya citado anteriormente, por un acercamiento en el tiempo de las fases propulsivas de piernas y brazos, debido un acortamiento de la fase de deslizamiento.

En ambas nadadoras, como se recoge en las Figuras 3 y 4 , existe una relación recíproca entre la frecuencia de ciclo y la distancia recorrida en cada ciclo. Esta relación corrobora los resultados ya encontrados en estudios previos (Craig y Pendergast, 1979; Chollet y col., 1996; Chollet y col., 1999; Soares y col., 1999).
En definitiva, podemos concluir que a mayor velocidad de desplazamiento, mayor pico de velocidad máxima intra-ciclo y frecuencia de ciclo. Por el contrario, la longitud de ciclo o duración de ciclo decrecerá debido a la reducción del tiempo de deslizamiento tras la acción propulsiva de piernas.

\section{Bibliografía}

1. Chengalur, S. N. \& Brown, P. (1992). Analy-sis of male and female olympics swimmers in the 200 meter events. Canadian Journal of Sports Sciences, 17, 104-109.

2. Chollet, D., Pelayo, P., Tourny, C. \& Syd-ney, M. (1996). Comparative analysis of $100 \mathrm{~m}$ and $200 \mathrm{~m}$ events in the four strokes in top level swimmers. Journal of Human Movement Studies, 31, 161-166.

3. Chollet, D., Tourny, C. et al. (1999). Evolution of coordination in flat breaststroke in relation to velocity. In K. L. Keskinen, P. V. Komi, A. P. Hollander (Eds). Biomechanics and Medicine in Swimming VIII (pp. 29-32). Jyväsklä, University of Jyväsklä.

4. Costill, D. L., D'acquisto, G. L. et al. (1987). Videocomputer assisted analysis of swimming technique. The Journal of Swimming Research, 3, 5-9.

5. Craig, A. B., Boomer, W. L. et al. (1988). Patterns of Velocity in Competitive Breaststroke Swimming. In B. E. Ungerechts, K. Wilke, K. Reischle (Eds). Swimming Science V, Vol. 18, (pp. 73-77). Bielefeld, Human Kinetics.

6. D'acquisto, L. J.,Costill D. L. et al. (1988). Breaststroke economy, skill and performance: study of breaststroke mechanics using a computer based «Velocity - Video System». The Journal of Swimming Research, 4, 9-13.

7. D'acquisto, L. J.,Costill D. L. (1998). Relationship between intra-cycle linear body velocity fluctuations, power and sprint breaststroke performance. The Journal of Swimming Research, 13, 814.

8. Kent, M. R. \& Atha, J. (1971). Selected critical transient body positions in breaststroke and their influence upon water resistance. First International Symposium on Biomechanics in Swimming. WaterPolo and Diving (pp. 119-126) Université Libre de Bruxelles : Bruxelles.

9. Kent, M. R. \& Atha, J. (1975). Intra-cycle kinematics and body configuration changes in the breaststroke. Second International Symposium on Biomechanics in Swimming (pp. 125-129). University Park Press: Bruselas.

10. Klauck, J., Daniel, K. et al. (1990). Velocity fluctuations in the breaststroke swimming cycle and their influence on average swimming speed. Paper presented at the Sixth International Symposium 
on Biomechanics and Medicine in Swimming. Liverpool.

11. Kolmogorov, S. V., Rumyantseva, O. A., Gordon B. J. \& Cappaert, J. M. (1997). Hydrodynamic characteristics of competitive swimmers of different genders and performance levels. Journal of Applied Biomechanics, 13, 88-97.
12. Kreighbaum, E. \& Barthels, K. M. (1990). Biomechanics: A qualitative approach for studying human movements. New York. Macmillan Publishing Company 